\title{
Intense Laser Field Effects on the Shallow-Donor Impurity States in Rectangular-Shaped Quantum Well Wires
}

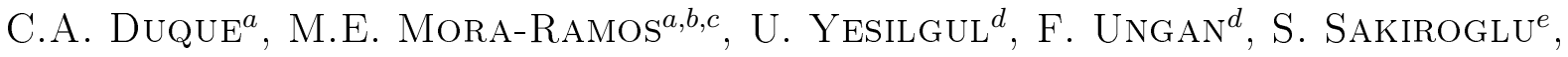 \\ E. KASAPOGLU ${ }^{d}$, H. SARI ${ }^{d}$ AND I. SÖKMEN ${ }^{e}$ \\ ${ }^{a}$ Grupo de Materia Condensada-UdeA, Instituto de Física, Facultad de Ciencias Exactas y Naturales \\ Universidad de Antioquia UdeA, Calle 70 No. 52-21, Medellín, Colombia \\ ${ }^{b}$ Facultad de Ciencias, Universidad Autónoma del Estado de Morelos, CP. 62209, México \\ ${ }^{c}$ Física Teórica y Aplicada, Escuela de Ingeniería de Antioquia, AA 7516, Medellín, Colombia \\ ${ }^{d}$ Cumhuriyet University, Physics Department, 58140 Sivas, Turkey \\ e Dokuz Eylül University, Physics Department, 35160 Buca, Izmir, Turkey \\ Using the effective mass and parabolic band approximations, the binding energy of a shallow donor impurity is \\ calculated in a $\mathrm{GaAs}-(\mathrm{Ga}, \mathrm{Al}) \mathrm{As}$ quantum well wire of rectangular transversal section, under the combined effects of \\ two independent axially-applied intense laser radiation fields and a static electric field oriented in the cross-section \\ plane. The lateral size of the rectangular cross-section is assumed to be larger than $10 \mathrm{~nm}$, in such a way that the \\ uncorrelated electron motion along the $x$ and $y$ directions can be considered uncoupled. The impurity-related states \\ are calculated by means of a variational procedure using a three-dimensional hydrogen-like trial wave function. \\ The intense laser field effects are introduced via the combination of the Floquet method for the laser-modified \\ confinement potential shape and the inclusion of a two-interaction centers model for the Coulombic coupling. It is \\ shown that, according to the polarization of the incident radiation, the quantum well wire can evolve from a single \\ 1D-heterostructure towards a configuration of two-well defined or four-well defined laser-induced parallel coupled \\ quantum well wires. The obtained results also show that the binding energy is strongly dependent on the impurity \\ position and on the strength of the intense laser field parameter.
}

DOI: $10.12693 /$ APhysPolA.125.198

PACS: 73.61.Ey, 78.67.De, 78.67.-n

\section{Introduction}

The influence of an intense high-frequency laser field on different physical properties of semiconductors has received some discussion and analysis in the literature $[1,2]$. The dressed atom approach was extended by Brandi et al. [3, 4] to treat the influence of the laser field upon a semiconductor system. The intense laser field (ILF) effects on the density of impurity states of shallow donors in a square, V-shaped, and inverse V-shaped quantum wells (QWs) have been studied by Niculescu et al. $[5,6]$. A theoretical study of the combined effects of intense high frequency laser and static magnetic fields on the binding and transition energies was developed by Niculescu et al. to investigate the ground and some excited states of an on-center hydrogenic donor in a cylindrically shaped GaAs quantum well wire (QWW) [7]. Besides, an investigation on the anisotropic optical absorption in QWWs, induced by high-frequency laser fields, has been also put forward [8].

A transition from single to double QW potential induced by ILF was revealed by Lima et al. [9]. Using the laser-dressed potential model in single QWs, the authors found the formation of a double-well potential structure in the regime $\alpha>L / 2$, where $L$ is the $\mathrm{QW}$ width and $\alpha$ is the laser-dressing parameter. The study of ILF effects has been extended to other heterostructures with or without the application of external probes such as applied electric and magnetic fields and hydrostatic pressure [10-13].

The present work concerns with the theoretical study of the effects of ILF on the hydrogenic-like shallow impurity states in QWWs with rectangular cross-section. The study includes the influence of independently applied laser radiation fields oriented along the horizontal and vertical directions in the $x y$ plane. In addition, we incorporate the influence of an in-plane-oriented static electric in order to investigate its effects on the impurity binding energy as well. The organization of the paper is as follows: in Sect. 2 we describe the theoretical framework. Section 3 contains the results and discussion, and finally, our conclusions appear in Sect. 4.

\section{Outlook of the theoretical model}

We shall deal with the effects of ILF on the binding energy of a shallow-donor impurity in a rectangular transversal section $\mathrm{GaAs}-\mathrm{Ga}_{0.7} \mathrm{Al}_{0.3} \mathrm{As}$ quantum wire in the presence of applied electric field. The calculation assumes the envelope-function and parabolic-band approximations. The choice for the electric field orientation is $\boldsymbol{F}=\left(F_{x}, F_{y}, 0\right)$. The transversal section of the wire is placed in the $x y$ plane and the $z$-axis is chosen along the main axis of the wire. In absence of laser field, the Hamiltonian for the electron-impurity system in a rectangular-section GaAs QWW under in-plane direction applied electric field is

$$
\begin{aligned}
H & =-\frac{\hbar^{2}}{2 m_{\mathrm{e}}}\left[\frac{\partial^{2}}{\partial x^{2}}+\frac{\partial^{2}}{\partial y^{2}}+\frac{\partial^{2}}{\partial z^{2}}\right]+V(x, y) \\
& +|e|\left(F_{x} x+F_{y} y\right)-\frac{e^{2}}{\varepsilon r}, \\
r & =\sqrt{\left(x-x_{0}\right)^{2}+\left(y-y_{0}\right)^{2}+z^{2}},
\end{aligned}
$$




$$
V(x, y)=V_{1}+V_{2} .
$$

In our model calculation we consider $V_{i}=V_{0}=225 \mathrm{meV}$ outside the wire region and zero into the wire region. Besides, $\left(x_{0}, y_{0}\right)$ labels the impurity position. This approximation is valid in quantum well wires with transversal section larger than $L_{x} \times L_{y}=10 \times 10 \mathrm{~nm}^{2}$, which corresponds mainly to the results reported in the present work. On the other hand, taking these dimensions also allows us to consider the laser-induced conduction band confining potential energy as a slowly varying function on the atomic length scale, which supports the use of the effective mass approximation. In order to include the non-resonant ILF effects, we adopt the Floquet method [14]. According to this formalism, the single particle $x$-dependent confinement potential is written as

$$
\begin{aligned}
& \left\langle V_{1}\right\rangle\left(x, \alpha_{x}\right)=\frac{V_{0}}{\pi} \sum_{m= \pm 1} \theta\left(\alpha_{x}+m x-L_{x} / 2\right) \\
& \times \arccos \left(\frac{L_{x} / 2-m x}{\alpha_{x}}\right), \\
& \alpha_{x}=\left(\frac{|e| A_{0}}{m_{\mathrm{e}} c \omega}\right)=\left(\frac{8 \pi e^{2} I}{m_{\mathrm{e}}^{2} c^{2} \omega^{4}}\right)^{1 / 2} .
\end{aligned}
$$

Here, $\alpha_{x}$ is the laser-dressing parameter (from now on ILF-parameter) [15]. In Eq. (2.2), $I$ and $\omega$ are, respectively, the average intensity and the frequency of the laser, $c$ is the speed of light, and $A_{0}$ is the amplitude of the vector potential associated with the incident radiation. There is an analogous expression for the confinement potential along the $y$-direction. Under the laser effects the last term of Eq. (2.1) - the one-center electronhole Coulomb interaction - must be replaced by a two-center Coulomb interaction as

$$
\begin{aligned}
& \left\langle V_{\mathrm{C}}\right\rangle(\boldsymbol{r}, \boldsymbol{\alpha})=-\frac{e^{2}}{2 \varepsilon}\left(\frac{1}{|\boldsymbol{r}+\boldsymbol{\alpha}|}+\frac{1}{|\boldsymbol{r}-\boldsymbol{\alpha}|}\right), \\
& \boldsymbol{\alpha}=\left(\alpha_{x}, \alpha_{y}, 0\right) .
\end{aligned}
$$

The ground state impurity wave function, and the corresponding energy, can be calculated via a RayleighRitz variational procedure. The energy of the electronimpurity system, $E$, is obtained after minimizing the Hamiltonian with the trial wave function. The impurity binding energy $\left(E_{\mathrm{b}}\right)$ is obtained from the definition: $E_{\mathrm{b}}=E_{0}-E$, where $E_{0}$ is the energy of the uncorrelated electron ground state in the QWW.

\section{Results and discussion}

We report the numerical outcome of the study for the case of a $\mathrm{GaAs}-\mathrm{Ga}_{0.7} \mathrm{Al}_{0.3} \mathrm{As}$ quantum well wire, as a prototypical system. The remaining set of parameters included in the calculations is: $\varepsilon=12.65$ and $m_{\mathrm{e}}=0.067 m_{0}$ (where $m_{0}$ is the free electron mass).

Figure 1 contains the calculated $1 s$-like electronimpurity binding energy as a function of the position of the donor atom along the $x$-direction for fixed $y_{0}$. The geometry of QWW's cross-section considered is the squared one, without the application of any external static electric field. The situation in which no ILF is applied to the system and $y_{0}=0$ shows the typical behavior of the binding energy for a QWW of square cross-section. A maximum is attained at the very center of the wire (on the $z$-axis) provided that, for symmetry reasons, the expected electron-impurity distance has its smaller value, thus leading to the strengthening of the Coulombic coupling.

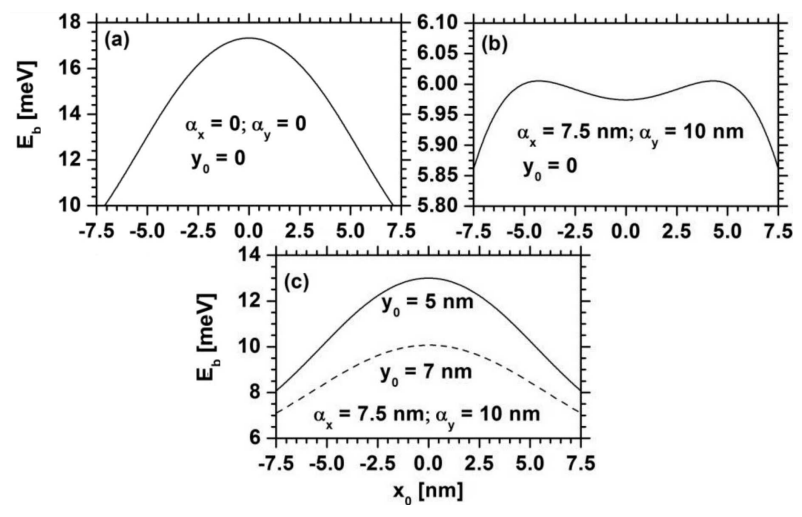

Fig. 1. Binding energy of a donor impurity in a GaAs$\mathrm{Ga}_{0.7} \mathrm{Al}_{0.3} \mathrm{As}$ quantum well wire with square transversal section as a function of the impurity position along the $x$-direction with $L_{x}=L_{y}=15 \mathrm{~nm}$ and $F_{x}=F_{y}=0$. The system is under the effects of intense laser field radiation with in plane-polarization of light: in (a) $\alpha_{x}=\alpha_{y}=0$, whereas in (b) and (c) the results are for $\alpha_{x}=7.5 \mathrm{~nm}$ and $\alpha_{y}=10 \mathrm{~nm}$. In (a) and (b) the impurity moves along the $x$-direction with $y_{0}=0$ whereas in (c) two different values of the $y_{0}$-coordinate have been considered.

Connecting the externally applied lasers along the $x$ and $y$-directions, keeping the same position for the impurity atom, causes an overall reduction of the binding energy due to the shifting of the electron uncorrelated wave function away from the origin that enlarges the expected electron-impurity distance (Fig. 1b). According to the explanation given in the previous section, the confining potential shape modifies due to the ILF influence. Along the $x$-direction it becomes a single- $\mathrm{V}$-shaped close to the origin - quantum well potential with larger effective width, given that $\alpha_{x}=L / 2$. On the other hand, the potential profile along the $y$-direction becomes that of a double quantum well, symmetrically positioned with respect to the origin. As a consequence, the uncorrelated electron wave function exhibits a local minimum centered at the origin (the reader is referred to observe Fig. 3c to see an analogous situation is depicted along the $y$-direction, although is different along the horizontal one).

Under the same configuration, shifting upwards the $y$ position of the impurity atom results in the recovery of the binding energy curve shape, Fig. 1b, but with reduced amplitude in comparison with the $E_{\mathrm{b}}$ shown in Fig. 1a. Now, the displacement of the donor atom brings closer the impurity to the electron; but only to one of the probability density maxima - the one associated with the split upper potential well. In consequence, the value of 
the binding energy reduces, as it is immediately apparent when the impurity vertical position is shifted further upward (dashed line in Fig. 1c).

In Fig. 2 we are presenting the results of the calculation of the electron-impurity binding energy as a function of both the in-plane angular orientations of the ILF (Fig. 2a) and a static electric field of intensity $F=50 \mathrm{kV} / \mathrm{cm}$ (Fig. 2b) — with no laser field applied. The donor atom locates at the origin (center of the rectangular cross-section). The system's cross-section has dimensions $L_{x}=15 \mathrm{~nm}$ and $L_{y}=20 \mathrm{~nm}$, and the maximum amplitude of the laser parameter components is $\alpha=5 \mathrm{~nm}$.
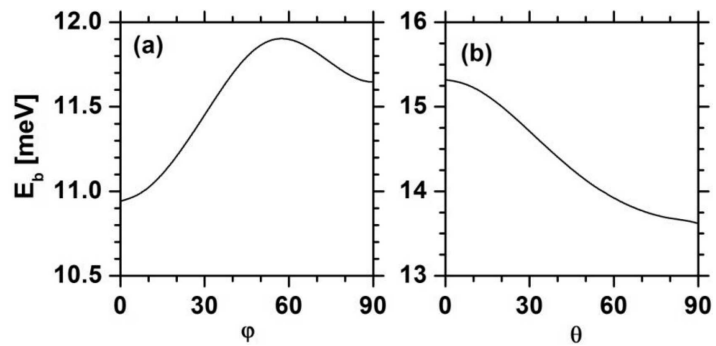

Fig. 2. Binding energy of a donor impurity in a GaAs- $\mathrm{Ga}_{0.7} \mathrm{Al}_{0.3} \mathrm{As}$ quantum well wire with rectangular transversal section as a function of the angle of the intense laser field in-plane polarization (a) and as a function of the angle on the in-plane applied electric field (b). The results are for $L_{x}=15 \mathrm{~nm}, L_{y}=20 \mathrm{~nm}$, and on-axis impurity $\left(x_{0}, y_{0}\right)$. In (a) $F_{x}=F_{y}=0$, $\alpha_{x}=\alpha \cos \theta, \alpha_{y}=\alpha|\sin \theta|$, and $\alpha=5 \mathrm{~nm}$. In (b) $\alpha_{x}=\alpha_{y}=0, F_{x}=F \cos \theta, F_{y}=F|\sin \theta|$, and $F=50 \mathrm{kV} / \mathrm{cm}$.

If there is only an in-plane-oriented applied laser radiation, $E_{\mathrm{b}}$ is a growing function of the angle $\theta$ until it reaches to a maximum at an angle of approximately $55^{\circ}$, and then starts decreasing. Here, one must keep in mind that, for a ILF parameter of only $5 \mathrm{~nm}$, the main effect is to effectively widen the conduction band QW along both horizontal and vertical directions. This effect is more pronounced along the $x$-direction given the smaller length of this rectangle side. As a consequence, the expected electron-impurity distance augments and the result is a lower value of $E_{\mathrm{b}}$, as noticed from Fig. 2a when $\theta=0$. The rise in the angle causes a competition between the reduction in width of the horizontal well and the increase in the effective potential well along the $y$-direction until there is a configuration that brings the maximum of the electron uncorrelated probability density closer to one of the Coulombic centers. There, we obtain the maximum of the binding energy seen in the figure. By further augmenting the angular amplitude we detect a decreasing variation; but this time, the rate of decrement is smaller, provided that the widening effect of the growing vertical component of the ILF is not as pronounced due to the larger length of this rectangle side.

In the case of an in-plane oriented static electric field in the zero laser regime, the consequence of the growth in the angular amplitude measured from the horizontal axis is to progressively diminish the value of the impurity binding energy - although the quantitative fall is pretty small. It is well known that the most prominent effect of the application of a static electric field on a QW is to provoke a displacement of the electron probability cloud towards the direction contrary to that of the field. This causes an increase of the expected distance between the electron and the Coulomb centers and, therefore, the reduction of $E_{\mathrm{b}}$. Since the size of the rectangular cross-section is larger in the $y$-direction, this effective separation is also greater and, as can be seen from Fig. $2 b$, the lowest value of the binding energy corresponds, precisely, to the case in which $\theta=90^{\circ}$. That is when the static electric field is applied along the $y$-axis.

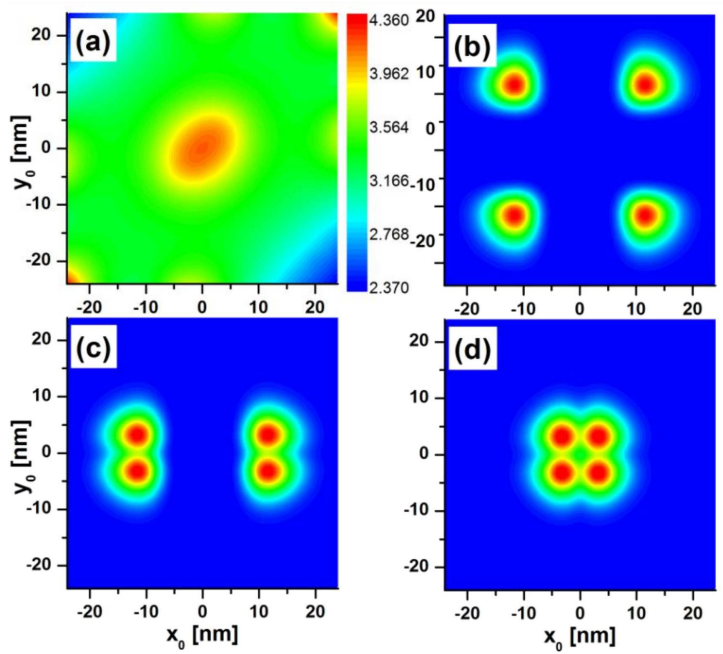

Fig. 3. (a) Binding energy of a donor impurity in a GaAs- $\mathrm{Ga}_{0.7} \mathrm{Al}_{0.3} \mathrm{As}$ quantum well wire with square transversal section as a function the in-plane impurity position $\left(x_{0}, y_{0}\right)$. In (b), (c), and (d) there is depicted the density of probability for the non-correlated ground state of the confined electron into the quantum well wire. In all the figures the results are for $L_{x}=L_{y}=15 \mathrm{~nm}$. Several values of the intense laser field in-plane polarization have been considered: $\alpha_{x}=\alpha_{y}=15 \mathrm{~nm}$ (a) and (b); $\alpha_{x}=15 \mathrm{~nm}$ with $\alpha_{y}=9 \mathrm{~nm}(\mathrm{c})$, and $\alpha_{x}=\alpha_{y}=9 \mathrm{~nm}(\mathrm{~d})$.

In Fig. 3a we are presenting a density plot of the impurity binding energy in a $\mathrm{GaAs}-\mathrm{Ga}_{0.7} \mathrm{Al}_{0.3} \mathrm{As}$ quantum well wire with square transversal section as a function of the in-plane impurity position $\left(x_{0}, y_{0}\right)$. The side dimensions and the values of the two components of the ILF vector parameter are all equal to $15 \mathrm{~nm}$. This figure has the aim of showing the actual complexity in the behavior of the binding energy when it is necessary to consider the presence and effect of two Coulomb centers in the problem.

Pulling off the donor impurity atom from the QWW heterostructure, the element of interest is the influence of the combined application of two in-plane ILFs on the electron states in a $\mathrm{GaAs}_{-}-\mathrm{Ga}_{0.7} \mathrm{Al}_{0.3} \mathrm{As}$ quantum well wire 
with square cross-section of side length $L=15 \mathrm{~nm}$. Restricting ourselves to take into account the conduction band ground state only, Fig. 3b-d shows the plots of the electron probability density for three different configurations of the intensities of laser radiations. Now, the value on the horizontal and vertical axes has nothing to do with the location of any Coulomb center. They only determine the in-plane position in the system.

In Fig. 3b we show the electron probability densities in the case when the two components of the ILF vector parameter are equal to $L$. Following the comments made in the theoretical framework, this configuration corresponds to the formation of double QW profiles along both the $x$ and $y$-directions. Consequently, we find a four-channel electron distribution, associated with the position of the laser-induced QW's.

If one of the components is smaller than $L$ (let us take $\alpha_{x}=15 \mathrm{~nm}$ and $\alpha_{y}=9 \mathrm{~nm}$ ) but still larger than $L / 2$, the configuration in the corresponding direction still corresponds to a double QW but the two potential wells are closer between each other. One sees that from Fig. 3c, where the same horizontal distance obtained for the probability maxima in Fig. 3b is kept, the vertical separation reduces significantly. This distribution then behaves as a two-channel one.

Finally, with the choice of $\alpha_{x}=\alpha_{y}=9 \mathrm{~nm}$, the two double QW profiles gather together, forming a single electron probability channel as seen from Fig. 3d. Although not depicted, choosing $\alpha_{x}=\alpha_{y}=L / 2$ would lead to the fusion of these four potential minima into a single one - placed at the square center - with an extremely localized electron wavefunction that resembles an actual one-dimensional electron distribution.

\section{Conclusions}

In this work we have studied the influence of intense laser and static electric fields on the electron states and the donor-impurity binding energy in a GaAs$\mathrm{Ga}_{0.7} \mathrm{Al}_{0.3} \mathrm{As}$ quantum well wire with rectangular cross-section. As a result of the calculation it is found that the binding energy is, in general, a complex function of the location of the impurity atom, as well as of the intensity and orientation of the two independent in-plane oriented intense laser fields. In general terms, the application of the laser radiations affects the problem of finding the binding energy by causing a reduction in the value of this quantity. This phenomenon is related with the appearance of two laser-induced Coulombic centers, and with the modification of the electron probability density due to the laser-induced change in the confining potential profile. Given the number of different input elements entering the calculation, we think that the problem of the donor impurity binding energy in this kind of systems deserves further consideration. On the other hand, we have shown that the combined use of two independent in-plane oriented intense laser radiation fields can be of usefulness for a selective switching of the electron probability distribution in quantum wires of rectangular cross-section, from single to twofold and even to four- fold coupled channels inside the wire. In this way, it is possible the mimicking of single, double and quadruple coupled one-dimensional electron threads within the structure. This possibility would also require an additional study.

\section{Acknowledgments}

M.E.M.R. thanks Mexican CONACYT for support through 2011-2012 sabbatical grant No. 180636, and research grant CB-2008-101777. He is also grateful to the Escuela de Ingeniería de Antioquia and the Universidad de Antioquia for hospitality during his sabbatical stay. This research was partially supported by Colombian Agencies: CODI-Universidad de Antioquia (Estrategia de Sostenibilidad 2013-2014 de la Universidad de Antioquia and the project: E01535-Efectos de la presión hidrostática y de los campos eléctrico y magnético sobre las propiedades ópticas no lineales de puntos, hilos y anillos cuánticos de GaAs- $(\mathrm{Ga}, \mathrm{Al}) \mathrm{As}$ y Si/SiO 2$)$, Facultad de Ciencias Exactas y Naturales-Universidad de Antioquia (CAD-exclusive dedication project 2012-2013), and by El Patrimonio Autónomo Fondo Nacional de Financiamiento para la Ciencia, la Tecnología y la Innovacion, Francisco José de Caldas. The authors are grateful to The Scientific and Technological Research Council of Turkey (TÜBİTAK) for a research grant (TÜBİTAK 109 T650). The work was done with the help of CENAPAD-SP, Brazil.

\section{References}

[1] L.C.M. Miranda, J. Phys. C, Solid State Phys. 9, 2971 (1976).

[2] O.A.C. Nunes, Solid State Commun. 45, 53 (1983).

[3] H.S. Brandi, A. Latgé, L.E. Oliveira, Phys. Status Solidi B 210, 671 (1998).

[4] H.S. Brandi, A. Latgé, L.E. Oliveira, Phys. Rev. B 70, 153303 (2004).

[5] E.C. Niculescu, L.M. Burileanu, A. Radu, Superlatt. Microstruct. 44, 173 (2008).

[6] E.C. Niculescu, A. Radu, M. Stafe, Superlatt. Microstruct. 46, 443 (2009).

[7] E.C. Niculescu, L.M. Burileanu, J. Optoelectron Adv. Mater. 9, 2713 (2007).

[8] E.C. Niculescu, L.M. Burileanu, A. Radu, A. Lupescu, J. Lumin. 131, 1113 (2011).

[9] F.M.S. Lima, M.A. Amato, O.A.C. Nunes, A.L.A. Fonseca, B.G. Enders, E.F. da Silva, Jr., J. Appl. Phys. 105, 123111 (2009).

[10] E. Kasapoglu, H. Sari, U. Yesilgul, I. Sökmen, J. Phys., Condens. Matter. 18, 6263 (2006).

[11] M. Santhi, A. John Peter, Eur. Phys. J. B 71, 225 (2009).

[12] C.A. Duque, E. Kasapoglu, S. Şakiroglu, H. Sari, I. Sökmen, Appl. Surf. Sci. 256, 7406 (2010).

[13] N. Eseanu, Phys. Lett. A 374, 1278 (2010).

[14] M. Gavrila, J.Z. Kaminski, Phys. Rev. Lett. 52, 613 (1984).

[15] H. Sari, E. Kasapoglu, I. Sökmen, Phys. Lett. A 311, 60 (2003). 